\title{
455.
}

\section{ON PLÜCKER'S MODELS OF CERTAIN QUARTIC SUREACES.}

[From the Proceedings of the London Mathematical Society, vol. III. (1869-1871), pp. 281-285. Read June 8, 1871.]

THE Society possesses a series of 14 wooden models of surfaces, constructed under the direction of the late Prof. Plücker, in illustration of the theory developed in his posthumous work, "Neue Geometrie des Raumes gegründet auf die Betrachtung der geraden Linie als Raumelemente," Leipzig, 1869. These all of them represent, I believe, Equatorial Surfaces; viz., models 1 to 8 represent cases of the 78 forms of equatorial surfaces "deren Breiten-Curven eine feste Axenrichtung besitzen," vol. II. pp. 352-363, the remaining models, Nos. 9-14, I have not completely identified. I propose to go into the theory only so far as is required for the explanation of the models.

In a "Complex," or triply infinite system of lines, there is in any plane whatever a singly infinite system of lines enveloping a curve; and if we attend only to the curves the planes of which pass through a given fixed line, the locus of these curves is a "complex surface." Similarly, there is through any point whatever a single infinite series of lines generating a cone; and if we attend only to the cones which have their vertices in the given fixed line, then the envelope of these cones is the same complex surface. In the case considered of a complex of the second degree, the curves and cones are each of them of the second order; the fixed line is a double line on the surface, so that (attending to the first mode of generation) the complete section by any plane through the fixed line is made up of this line twice, and of a conic; the surface is thus of the order $4:$ it is also of the class 4 ; the surface has, in fact, the nodal line, and also 8 nodes (conical points), and we have thus a reduction $=32$ in the class of the surface.

In the particular case where the nodal line is at infinity, the complex surface becomes an equatorial surface; viz., (attending to the first mode of generation) we have 
here a series of parallel planes each containing a conic, and the locus of these conics is the equatorial surface.

It is convenient to remark that, taking $a, b, h$ to be homogeneous functions of $(x, w)$ of the order $2 ; f, g$ of the order 1 ; and $c$ of the order 0 (a constant); then the equation of a complex surface (see vol. I. p. 162) is

$$
\left|\begin{array}{llll} 
& y, & z, & 1 \\
y, & a, & h, & g \\
z, & h, & b, & f \\
1, & g, & f, & c
\end{array}\right|=0
$$

and that, writing $w=1$, or considering $a, h, b ; f, g ; c$ as functions of $x$ of the orders $2,1,0$ respectively, we have an equatorial surface.

A particular form of equatorial surface is thus $b c y^{2}+c a z^{2}+a b=0$, or taking $c=1$, this is $b y^{2}+a z^{2}+a b=0$, where $a, b$ are quadric functions of $x$.

The surface is still, in general, of the fourth order: it may however degenerate into a cubic surface, or even into a quadric surface; the last case is however excluded from the enumeration. The section by any plane parallel to that of $y z$ is a conic; the section by the plane $y=0$ is made up of the pair of lines $a=0$, and of the conic $z^{2}+b=0$; that by the plane $z=0$ is made up of the pair of lines $b=0$, and of the conic $y^{2}+a=0$; the last-mentioned planes may be called the principal planes, and the conics contained in them principal conics. The surface is thus the locus of a variable conic, the plane of which is parallel to that of $y z$, and which has for its vertices the intersections of its plane with the two principal conics respectively. And we have thus the particular equatorial surfaces considered by Pliicker, vol. II. pp. 346-363 (as already mentioned), under the form

$$
\frac{y^{2}}{E x^{2}+2 U x+C}+\frac{z^{2}}{F x^{2}-2 R x+B}+1=0,
$$

and of which he enumerates 78 kinds; viz., these are

1 to 17 Principal conics each proper.

18 „29 One of them a line-pair.

30 „32 Each a line-pair.

33 „ 39 Principal conics, each proper, but having a common point.

40 " 43 One of them a line-pair, its centre on the other principal conic.

44. „61 One principal conic a parabola.

62 „73 One principal conic a pair of parallel lines.

74 „76 Principal conics each a parabola.

77 and 78 Principal conics, one of them a parabola, the other a pair of parallel lines. 
The models 1 to 8 correspond hereto, as follows:

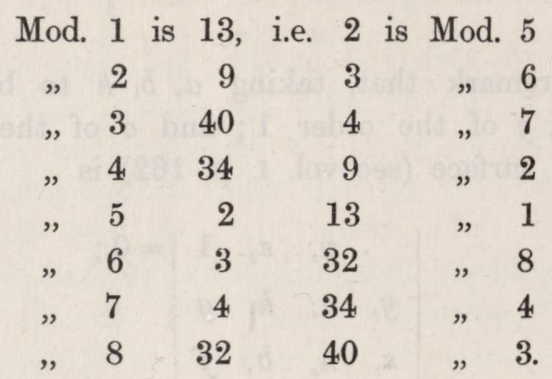

Mod. 5 is 2: the form of the equation is here

$$
\frac{y^{2}}{l^{2}\left[(x-\alpha)^{2}+\beta^{2}\right]}-\frac{z^{2}}{l^{\prime 2}\left[\left(x-\alpha^{\prime}\right)^{2}+\beta^{\prime 2}\right]}=1
$$

viz., the principal conics are one of them a hyperbola, the other imaginary; hence the generating conic has always two, and only two, real vertices, viz., it is always a hyperbola : there are no real lines.

Mod. 6 is 3 : the form of the equation is

$$
\frac{y^{2}}{l^{2}\left[(x-\alpha)^{2}+\beta^{2}\right]}+\frac{z^{2}}{l^{\prime 2}\left[\left(x-\alpha^{\prime}\right)^{2}+\beta^{\prime 2}\right]}=1
$$

viz., the principal conics are each of them a hyperbola; the generating conic has four real vertices, viz., it is always an ellipse: there are no real lines.

Mod. 7 is 4 : the form of the equation is

$$
\frac{y^{2}}{l^{2}(x-\gamma)(x-\delta)}+\frac{z^{2}}{l^{\prime 2}\left[\left(x-\alpha^{\prime}\right)^{2}+\beta^{\prime 2}\right]}+1=0 .
$$

The principal conics are one of them an ellipse, the other imaginary; for values of $x$ between $\gamma$ and $\delta$, the variable conic has two real vertices or it is a hyperbola; for any other values it is imaginary, so that the surface lies wholly between the planes $x=\gamma, x=\delta$ : the surface contains the real lines $y=0, x=\gamma$ and $y=0, x=\delta$.

Mod. 2 is 9 : the form of the equation is

$$
\frac{y^{2}}{l^{2}(x-\gamma)(x-\delta)}+\frac{z^{2}}{l^{\prime 2}\left(x-\gamma^{\prime}\right)\left(x-\delta^{\prime}\right)}+1=0
$$

where, say the values $\gamma, \delta$ lie between the values $\gamma^{\prime}, \delta^{\prime}$ : the principal conics are each of them an ellipse, the vertices (on the axis or line $y=0, z=0$ ) of the one ellipse lying between those of the other ellipse. The variable conic for values of $x$ between $\gamma, \delta$ has four real vertices, or it is an ellipse; for values beyond these limits, but within the limits $\gamma^{\prime}, \delta^{\prime}$-say from $\gamma$ to $\gamma^{\prime}$, and from $\delta$ to $\delta^{\prime}$-there are two real vertices, or the conic is a hyperbola; and for values beyond the limits $\gamma^{\prime}, \delta^{\prime}$, the variable conic is imaginary. 
There are four real lines $(y=0, x=\gamma),(y=0, x=\delta),\left(z=0, x=\gamma^{\prime}\right),\left(z=0, x=\delta^{\prime}\right)$. The surface consists of a central pillow-like portion, joined on by two conical points to an upper portion, and by two conical points to an under portion, the whole being included between the planes $x=\gamma^{\prime}, x=\delta^{\prime}$.

Mod. 1 is 13: the form of the equation is

$$
\frac{y^{2}}{l^{2}(x-\gamma)(x-\delta)}-\frac{z^{2}}{l^{\prime 2}\left(x-\gamma^{\prime}\right)\left(x-\delta^{\prime}\right)}+1=0 ;
$$

the values $\gamma^{\prime}, \delta^{\prime}$ lying between $\gamma, \delta$; the principal conics are one of them a hyperbola, the other an ellipse, the vertices (on the axis or line $y=0, z=0$ ) of the hyperbola lying between those of the ellipse.

The variable conic, for values of $x$ between $\gamma^{\prime}, \delta^{\prime}$, has two real vertices, or it is a hyperbola; for the values, say, from $\gamma^{\prime}$ to $\gamma$, and $\delta^{\prime}$ to $\delta$, there are four real vertices, or the conic is an ellipse; for values beyond the limits $\gamma, \delta$, there are two real vertices, and the conic is a hyperbola. There are the four real lines $(y=0, x=\gamma),(y=0, x=\delta)$, and $\left(z=0, x=\gamma^{\prime}\right),\left(z=0, x=\delta^{\prime}\right)$. The surface consists of 8 portions joined to each other by 8 conical points, but the form can scarcely be explained by a description.

Mod. 8 is 32 : the form of the equation is

$$
\frac{y^{2}}{l^{2}(x-\gamma)^{2}}+\frac{z^{2}}{l^{2}\left(x-\gamma^{\prime}\right)^{2}}=1 ;
$$

viz., the principal conics are each of them a line-pair, the variable conic is always an ellipse.

There are the two real nodal lines $(y=0, x=\gamma)$ and $\left(z=0, x=\gamma^{\prime}\right)$, each of these being in the neighbourhood of the axis crunodal, and beyond certain limits acnodal; the surface is a scroll, being, in fact, the well-known surface which is the boundary of a small circular pencil of rays obliquely reflected, and consequently passing through two focal lines.

Mod. 4 is 34 : the equation is

$$
\frac{y^{2}}{l^{2}(x-\gamma)(x-\delta)}+\frac{z^{2}}{l^{\prime 2}\left(x-\gamma^{\prime}\right)(x-\delta)}+1=0,
$$

where $x=\delta$ is not intermediate between the values $x=\gamma$ and $x=\gamma^{\prime}$; say the order is $\delta, \gamma, \gamma^{\prime}$. The surface is thus a cubic surface; the principal conics are ellipses having on the axis a common vertex at the point $x=\delta$, and the remaining two vertices on the same side of the last-mentioned one. The variable conic for values between $\delta$ and $\gamma$ has four real vertices, or it is an ellipse; for values between $\gamma$ and $\gamma^{\prime}$ two real vertices, or it is a hyperbola; and for values beyond the limits $\delta, \gamma^{\prime}$ it is imaginary. There are on the surface the two real lines $(y=0, x=\gamma)$ and $\left(z=0, x=\gamma^{\prime}\right)$. The surface consists of a finite portion joined on by two conical points to the remaining portion. 
Mod. 3 is 40 : the form of equation is

$$
\frac{y^{2}}{l^{2}(x-\gamma)(x-\delta)}+\frac{z^{2}}{l^{\prime 2}(x-\delta)^{2}}+1=0 .
$$

The surface is thus a cubic surface; the principal conics are, one of them an ellipse, the other a pair of imaginary lines intersecting on the ellipse; for values of $x$ between $\gamma$ and $\delta$, the variable conic has thus two real vertices, and it is a hyperbola; for values beyond these limits it is imaginary, and the whole surface is thus included between the planes $x=\gamma$ and $x=\delta$. There are the two real lines $(y=0, x=\gamma)$ and $(z=0, x=\delta)$.

Taking $l^{2}=l^{\prime 2}=1$, the surface is

$$
\frac{y^{2}}{(x-\gamma)(x-\delta)}+\frac{z^{2}}{(x-\delta)^{2}}+1=0
$$

which is a particular case of the parabolic cyclide.

As already mentioned, I have not completely identified the remaining models 9 to 14 , but I will say a few words about them.

The equatorial surfaces, not included in the preceding 78 cases, Plücker distinguishes (voi. II. p. 363) as "gedrehte" or "tordirte," say as twisted equatorial surfaces; the equation of such a surface is

where

$$
b y^{2}+2 h y z+a z^{2}+a b-h^{2}=0,
$$

$$
\begin{aligned}
& b=F x^{2}-2 R x+B, \\
& a=E x^{2}+2 U x+C, \\
& h=K x^{2}-O x-G(\text { or in particular }=-O x-G) .
\end{aligned}
$$

Mod. 13 is such a surface, being a twisted form of model 2.

Mod. 9 and Mod. 14 belong, I think, to the case $a=0$; viz., the form of the equation is $b y^{2}+2 h y z-h^{2}=0$. The variable conic is a hyperbola, the direction of one of the asymptotes being constant (vol. II. p. 368).

There are moreover (p. 372) equatorial surfaces in which the variable conic is always a parabola, and where there are on the surface four real or imaginary lines.

Mods. 10, 11, and 12 seem to represent such surfaces. 